\title{
Surgical Robotic Arm with Pneumatic Semi-active Joints for Load Reduction on Tool-insertion Port
}

\author{
Koki Aizawa, ${ }^{1}$ Masao Kanazawa, ${ }^{2}$ Daisuke Haraguchi, ${ }^{3}$ and Kotaro Tadano ${ }^{2,3 *}$ \\ ${ }^{1}$ Department of Mechanical Engineering, Tokyo Institute of Technology, \\ 4259 Nagatsuta-cho, Midori-ku, Yokohama City 226-8503, Japan \\ ${ }^{2}$ RIVERFIELD Inc., 20 Samon-cho, Shinjuku-ku, Tokyo 160-0017, Japan \\ ${ }^{3}$ Institute of Innovative Research, Tokyo Institute of Technology, \\ 4259 Nagatsuta-cho, Midori-ku, Yokohama City 226-8503, Japan
}

(Received November 18, 2019; accepted December 10, 2019)

Keywords: surgical robot, laparoscopic surgery, remote center of motion, pneumatic actuator, force control

In minimally invasive robotic surgeries, insertion ports become the fulcrum of surgical instruments such as forceps. Misalignment between the insertion port and the remote center of motion (RCM) of the robotic arm or external forces on the tip of its forceps generate undesirable forces and friction between the port and the forceps. It not only damages the port but also worsens position control performance. In this study, we developed a pneumatic surgical robotic arm that does not require the positioning of its RCM on the insertion port. Force-compensatable joints that are compliant with the joint position and have the ability to output torque are employed. We call these joints "semi-active joints", and we realize them with pneumatic actuators with high backdrivability. The posture of the robotic arm with the semi-active joints is decided passively by the constraint of the insertion port. In addition, the robotic arm can compensate for the weight of its forceps and external forces to reduce the load on the insertion port. With the prototype surgical robotic arm system, the position control of the robotic forceps was performed, and we evaluated the accuracy of the arm's movement and the forces exerted on the insertion port. The experimental results showed that the load on the insertion port was decreased by $74 \%$, when using semi-active joints.

\section{Introduction}

Recently, laparoscopic surgical technology using a surgical robot has markedly developed. In laparoscopic surgical operation, the surgical instruments (i.e., forceps) are inserted into the insertion port. Thus, the robot is required to move while maintaining its remote center of motion (RCM) using the insertion port as a fulcrum. There are mainly three methods of dealing with the insertion port.

The first method is that the robot mechanically maintains a fixed point by the RCM mechanism. It has been adopted in many surgical robots such as the da Vinci Surgical System ${ }^{(1)}$ developed by Intuitive Surgical and IBIS IV ${ }^{(2)}$ by Tadano et al. With this RCM mechanism,

*Corresponding author: e-mail: tadano.k.aa@m.titech.ac.jp

https://doi.org/10.18494/SAM.2020.2713 
the robot can easily work, maintaining its RCM. Before the start of a surgery, however, it is necessary to precisely align the RCM with the insertion port for instruments on the patient's body, which is troublesome during the setup. Moreover, the misalignment between the RCM and the insertion port during the setup or certain operations damages the insertion port and increases harmful friction between the insertion port and the instruments. It degrades the controlled position performance of the robot.

The second method requires that a surgical robot has redundant degrees of freedoms (DOFs) to maintain the RCM by control. An example of such a robotic arm is DLR MIRO ${ }^{(3)}$ developed by Hagn et al. Sadeghian et al. conducted research on robots with redundant DOFs. ${ }^{(4)}$ Owing to the redundant DOFs, the RCM can be set in a wide range in a reachable area, and the robot can realize its movement. However, when the joints of the robot are highly rigid or have a low backdrivability, forces may be exerted on the insertion port owing to the misalignment between the RCM and the insertion port. Also, if the insertion port is misaligned and its position is misrecognized, it is difficult to follow the reference trajectory of the instrument position without applying a load to the insertion port.

The third method requires that a surgical robotic arm has some passive joints at its wrist. Owing to the physical constraint of the insertion port, the joints move passively so that the instrument pivots about its fixed point. Examples of surgical robotic arms that use passive joints are ZEUS developed by Computer Motion ${ }^{(5)}$ and MicroHand ${ }^{(6)}$ developed by Wang et al. Because the posture of the robotic arm is determined passively by the constraint of the insertion port, the robot does not require precise alignment of RCM, and no forces are exerted on the insertion port even when misalignment occurs. In addition, algorithms for insertion port estimation have been studied to deal with misalignment. ${ }^{(7,8)}$ However, the arm cannot compensate for the weight of its forceps and external forces owing to its passive joints. As a result, forces are exerted on the insertion port and friction increases.

Robots that can actively maintain its RCM such as robots with the RCM mechanism and robots with redundant DOFs require precise alignment and cannot deal with any misalignment. On the other hand, robots with passive joints can deal with misalignments, but cannot compensate for the weight of its forceps and external forces. Therefore, in this study, we aim to develop a surgical robotic arm that can deal with misalignment and can compensate for the weight of its forceps and external forces. We used backdrivable pneumatic actuators instead of passive joints and fabricated the surgical robotic arm having a suitable number of DOFs for the robot to maintain its RCM passively. We call these backdrivable joints "semi-active joints", which are not controlled by position feedback, but by torque to compensate for the weight of forceps and external forces. With this structure, the surgical robotic arm does not require the precise alignment of its RCM with the insertion port, thereby eliminating the need for the troublesome setup and reducing the force exerted on the patient's body. It also reduces friction between the insertion port and the forceps, which enables better control of the forceps.

This paper is structured as follows. In Sect. 1, we describe recent surgical robots and classify them by their structure. In Sect. 2, we describe the surgical robotic arm we developed, its structure, and its control system. In Sect. 3, we describe the experiment we conducted to evaluate our robot. In Sect. 4, we discuss the robots we developed and our future works. 


\section{Materials and Methods}

\subsection{Development of surgical robotic arm with semi-active joints}

\subsubsection{Structure of the robotic arm}

The surgical robot we developed is shown in Fig. 1. The robot is divided into the holder arm and the forceps. The holder arm has 6 DOFs and the forceps has 2 DOFs. Therefore, the entire robot functions have 8 DOFs, but the arm is under constraint by the insertion port. Thus, it has 6 DOFs for the abdomen. All of the joints are driven by pneumatic actuators. With pneumatic actuators, we could make the robot compact in size because the mass-to-power ratio of the pneumatic actuators is very high. In addition, they have high backdrivability, which enables the robot to estimate the external force. The pneumatic driving system makes the robot soft, which realizes more delicate tissue manipulation with respect to forces. These actuators have many advantages for a surgical robot. This is why we adopt pneumatic actuators for our surgical robot. $q_{1}, q_{4}, q_{5}$, and $q_{6}$ are driven by pneumatic vane motors and $q_{2}$ and $q_{3}$ by pneumatic cylinders with parallel links. $q_{7}$ and $q_{8}$ are driven by wire from cylinders in its forceps driver.

Although all of the joints are driven by pneumatic actuators, $q_{4}$ and $q_{5}$ are especially used as semi-active joints. Owing to the arrangement of the semi-active joints, the posture of the robotic arm can be decided passively by the constraint of the insertion port. Also, as shown in Fig. 2, owing to the properties of parallel links, the rotational axis of $q_{4}$ always has an angle of $45^{\circ}$ to a horizontal surface. It enables the arm to avoid its singular points during surgical operation. Furthermore, all of the joints have rotary encoders, and we can use the angles of the joints to obtain its posture.

\subsubsection{Gimbal mechanism of semi-active joints}

We developed semi-active joints using highly backdrivable pneumatic vane motors. As shown in Fig. 2, the joints are arranged at $q_{4}$ and $q_{5}$, and work as gimbal joints that can hold forceps. We made these joints compact, as shown in Fig. 3. For $q_{4}$ and $q_{5}$, single-vane motors

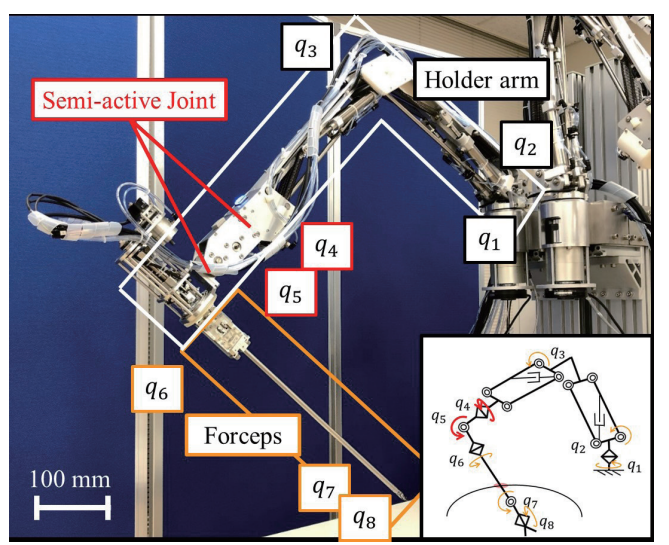

Fig. 1. (Color online) Structure of the surgical robotic arm with semi-active joints. 


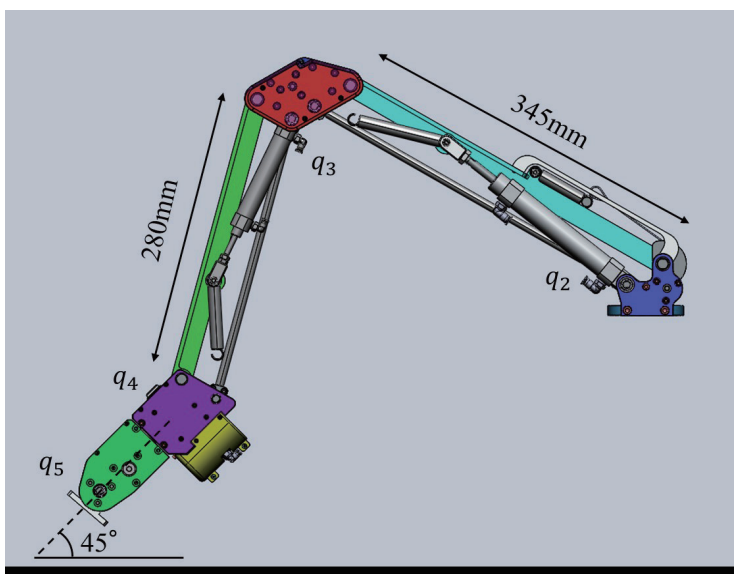

Fig. 2. (Color online) Parallel link mechanisms of the surgical robotic arm.

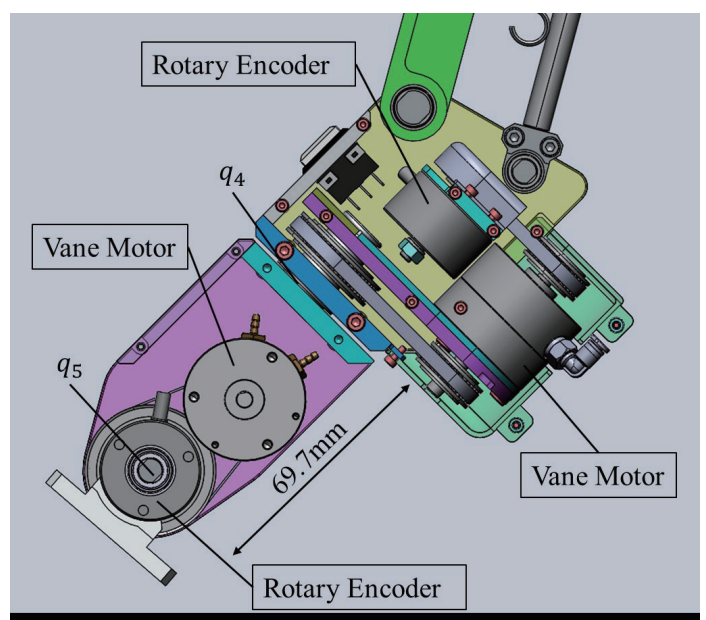

Fig. 3. (Color online) Structure of the semi-active joints.

(Kuroda; PRNA3S-270-45) were used with belt-pulley drive mechanisms whose reduction ratios are 1.56 and 1.88, respectively. These $q_{4}$ and $q_{5}$ joints can output torques of about 590 and $710 \mathrm{~N} \cdot \mathrm{mm}$, respectively, when the supply pressure is $0.6 \mathrm{MPa}$. The joints are not controlled by the position feedback system and they are not stiff. Therefore, the posture of the joint is determined passively. Semi-active joints also function as actuators, and this structure of the joints enables the output of torque to compensate for the weight of the forceps and forces exerted on the tip of the forceps.

\subsection{Control method}

\subsubsection{Control system}

Here we describe the control system of our robot. A block diagram of the control system with normal stiff joints $q_{1}, q_{2}, q_{3}$, and $q_{6}$ is shown in Fig. 4 , and that of semi-active joints $q_{4}$ and $q_{5}$ is shown in Fig. 5. I.K. means the calculation of inverse kinematics, and PID means the proportional-integral-derivative controller. The joint torque controller is the torque controller of the pneumatic actuators.

For the normal stiff joints, we obtain references of the angle of each joint by calculating inverse kinematics and perform position control by the PID control system. On the other hand, for the semi-active joints, we do not perform position control for stiff joints, but perform feedforward control and torque control to compensate for the weight of the forceps, the dynamics of the arm and the forceps, and external forces. In this paper, although the arm compensates only for the weight of the forceps and the dynamics, the arm is expected to output torque to have all external forces on forceps and reduce the forces exerted on the insertion port. 


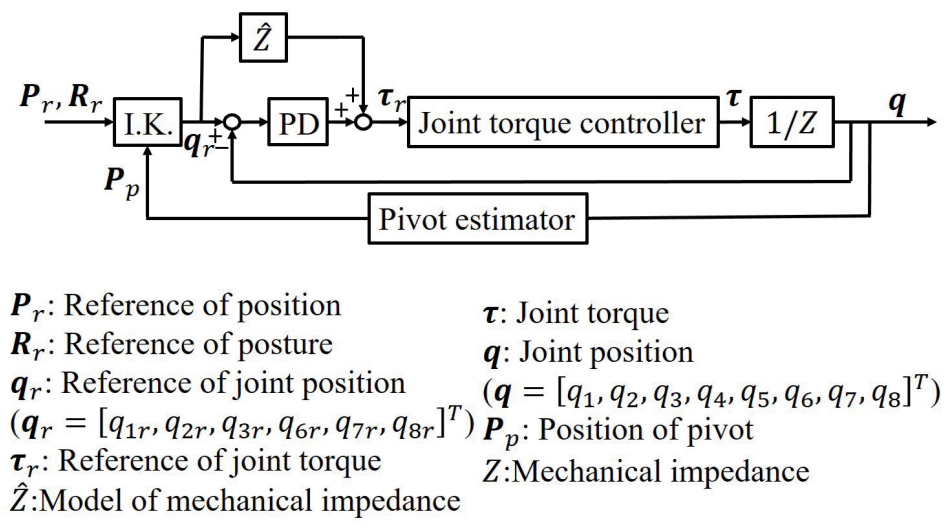

Fig. 4. Block diagram of control system with normal stiff joints.

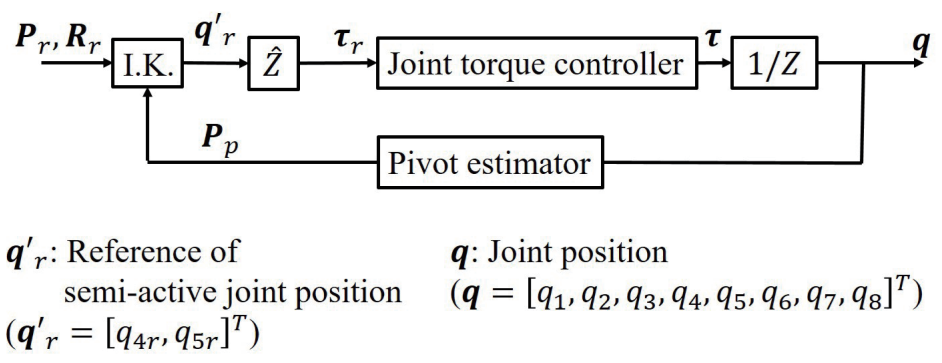

Fig. 5. Block diagram of control system with semi-active joint.

\subsubsection{Inverse kinematics}

To control the position of the tip of the forceps, the solution of inverse kinematics is presented. This robot has 8 redundant DOFs when considering the arm, semi-active joints, and forceps. However, since it is constrained by the insertion port with 2 DOFs, the inverse kinematics can be solved if the position of the insertion port is known. As shown in Fig. 1, the robot is divided into the robotic arm and the forceps. Here, we show the method of solving the inverse kinematics on the basis of the position of the insertion port and by dividing calculation into the outside and inside of the insertion port. As shown in Fig. 6, the world coordinate $\{\mathrm{O}\}$ is defined, placing its origin on the first joint, and the abdominal coordinate $\{\mathrm{T}\}$ is also defined, placing its origin on the insertion port. Then, the joint angles of the forceps in the frame $\{\mathrm{T}\}$ are $q_{t 1}-q_{t 6}$, as shown in Fig. 6(b). The procedure of inverse kinematics calculation is shown in Fig. 7. $P_{r}$ and $R_{r}$ mean the position and the posture of the tip, respectively. $P_{6}$ and $R_{6}$ are the position and posture of the joint $q_{6}$. The superscripts $\mathrm{O}$ and $\mathrm{T}$ indicate the frames $\{\mathrm{O}\}$ and $\{\mathrm{T}\}$, respectively.

In this way, by dividing the calculation into the outside and inside of the insertion port, inverse kinematics of this robot can be solved. $q_{4}$ and $q_{5}$ in the robot are semi-active and their position feedback controls are not applied. However, $q_{4}$ and $q_{5}$ are used for calculation of feedforward control. Thus, it is necessary to obtain $q_{1}-q_{8}$. Considering this method, we can 


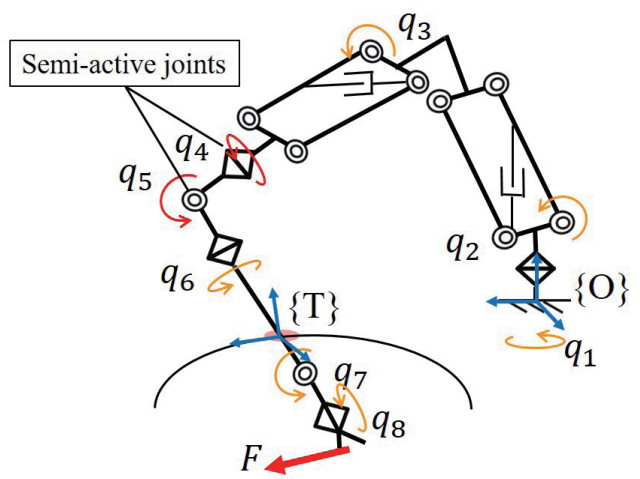

(a)

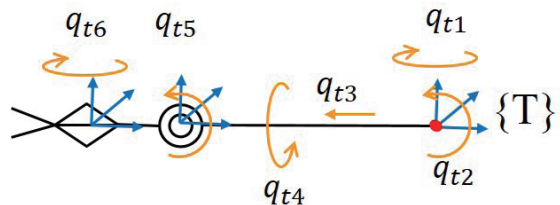

(b)

Fig. 6. (Color online) Joint position of the robotic arm.

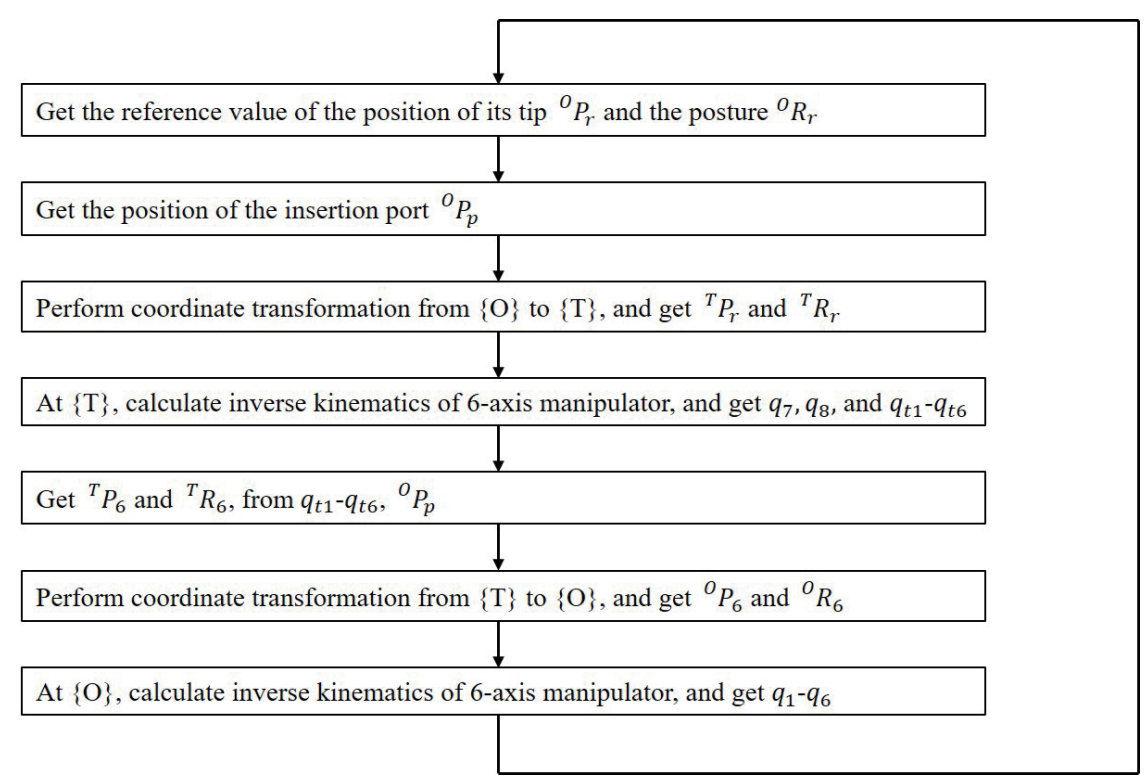

Fig. 7. Algorithm of inverse kinematics.

separately deal with the inverse kinematics of its arm and that of forceps. Therefore, if the arm or the forceps changes, we do not have to change the entire algorithm, but change only the inverse kinematics of the changed part. This algorithm can be generally used for robots having the same structure.

This algorithm requires the position of the insertion port, and the proposed robot is expected to be used when its position is unknown. Thus, the insertion port has to be estimated or measured. Estimation methods for the insertion port have been researched. ${ }^{(7,8)}$ In this study, we easily estimated the insertion port using the velocity, angular velocity, and geometric information of the wrist of the arm. 


\subsubsection{Joint torque controller of pneumatic system}

The joint torque controller is a control system that outputs the desired torque. Since this robot is driven by pneumatic actuators, we explain the pneumatic torque controller mechanism based on a pneumatic pressure feedback loop. Figure 8 shows a pneumatic circuit for a 1-axis pneumatic actuator. The air flow of the actuator is controlled by a spool-type servo valve with 5 ports. The pressure within the actuator is measured by pressure sensors. We can calculate driven power from the pressure difference in the valve.

Pneumatic actuators have nonlinear characteristics owing to the compressibility of air, but this can be improved by the inner pressure loop in the control system. ${ }^{(9)}$ As shown in Fig. 9, we designed the closed loop system of value equivalent to differential pressure, using it as the "joint torque controller".

\section{Experiment and Results}

\subsection{Experiment on control to reduce external forces at insertion port}

We conducted an experiment to evaluate the effectiveness of the proposed robotic arm. With its RCM kept passively, we examined whether the arm can reduce external forces on the insertion port by using semi-active joints. Figure 10 shows the experimental setup. We used an experimental device as a model of insertion port. The insertion port is on a free gimbal joint

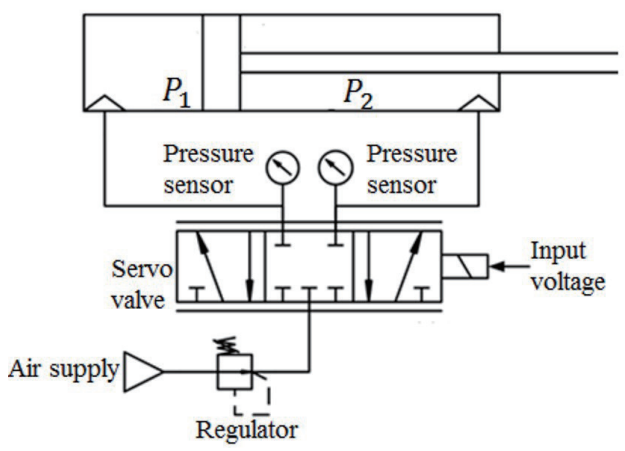

Fig. 8. Pneumatic circuit diagram of valve and actuator.

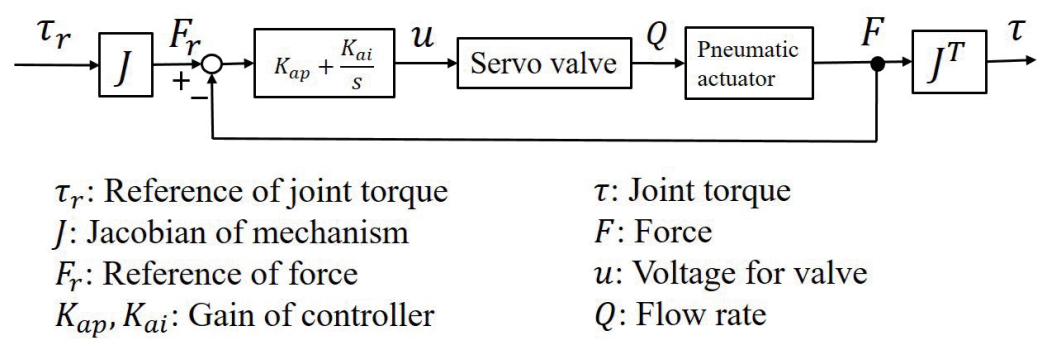

Fig. 9. Block diagram of pneumatic joint torque controller. 


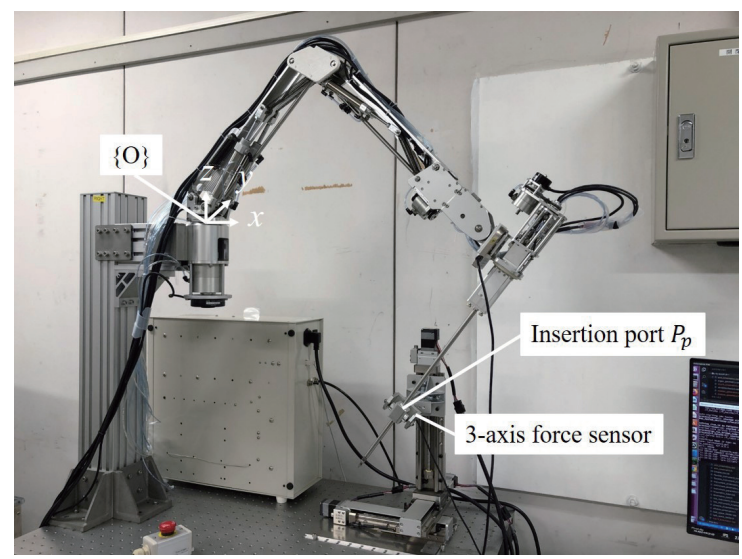

Fig. 10. (Color online) Experimental setup.

that can freely rotate in the roll and pitch directions. At the base of the gimbal, a 3-axis force sensor is placed. Using this device, we measured the external forces on the insertion port while the arm was working.

A circular trajectory with a radius of $30 \mathrm{~mm}$ was given as the target trajectory for the forceps. The frequency of the trajectory is $0.1 \mathrm{~Hz}$. Origin of the coordinate is put on the joint $q_{1}$, as shown in Fig. 10. Then, the position of the insertion port $P_{p}$ can be expressed as $P_{p}=[445 \mathrm{~mm},-30 \mathrm{~mm},-338 \mathrm{~mm}]^{\mathrm{T}}$. We investigated the accuracy of the movement of the forceps and the forces applied to the insertion port during the work. The experiment was conducted under two conditions: completely passive and semi-active gimbal joints of the robotic arm. In this experiment, passive means that no driving force is generated from the semi-active joints. Pneumatic actuators have high backdrivability. Even when there is slight friction and no driving torque is applied, the actuator works similarly to a passive joint. The conditions of the experiment are shown in Table 1.

\subsection{Experimental results}

Figure 11 shows the trajectory of the tip of the forceps. RMSE are calculated from the time series tracking error. RMSE of the trajectory with passive joints is $2.40 \mathrm{~mm}$, and that with semi-active joints is $1.92 \mathrm{~mm}$. It is found that followability of the tip position is better when the gimbal joint of the wrist of the arm is semi-active than when the joint is passive. Figure 12 shows forces exerted on the insertion port measured by the force sensor on the experimental device. The average magnitude of forces during the work is shown in Table 2. The magnitude of the external force is decreased by $74 \%$ when the semi-active joints are used, compared with that when the passive joints are used. Figure 12 also shows that the load on the insertion port is smaller when the semi-active joints are used. This is because the weight of the forceps and the friction generated in the joints can be compensated for by feedforward control of the semiactive joints. Figures 13 and 14 show the output torque of the semi-active and passive joints, respectively. It can be confirmed from Fig. 13 that torques were output from the semi-active joints as needed. 
Table 1

Experimental conditions.

\begin{tabular}{lc}
\hline Joint controller & Passive/Semi-active \\
\hline Reference trajectory & $(x-310)^{2}+y^{2}=30^{2} \mathrm{~mm}^{2}, z=-412 \mathrm{~mm}$ \\
\hline
\end{tabular}

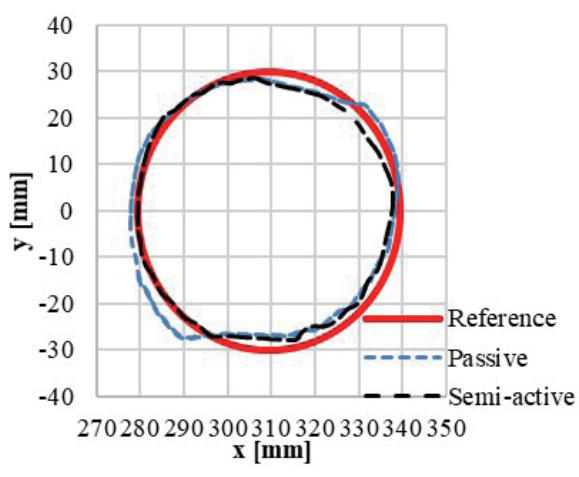

(a)

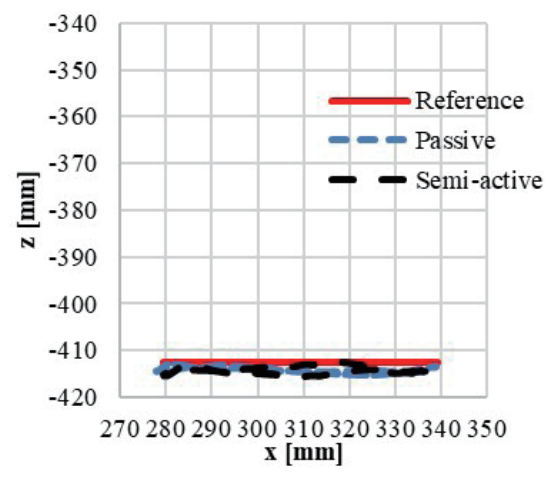

(b)

Fig. 11. (Color online) Trajectory of the tip of the forceps. (a) $x-y$ and (b) $x-z$.
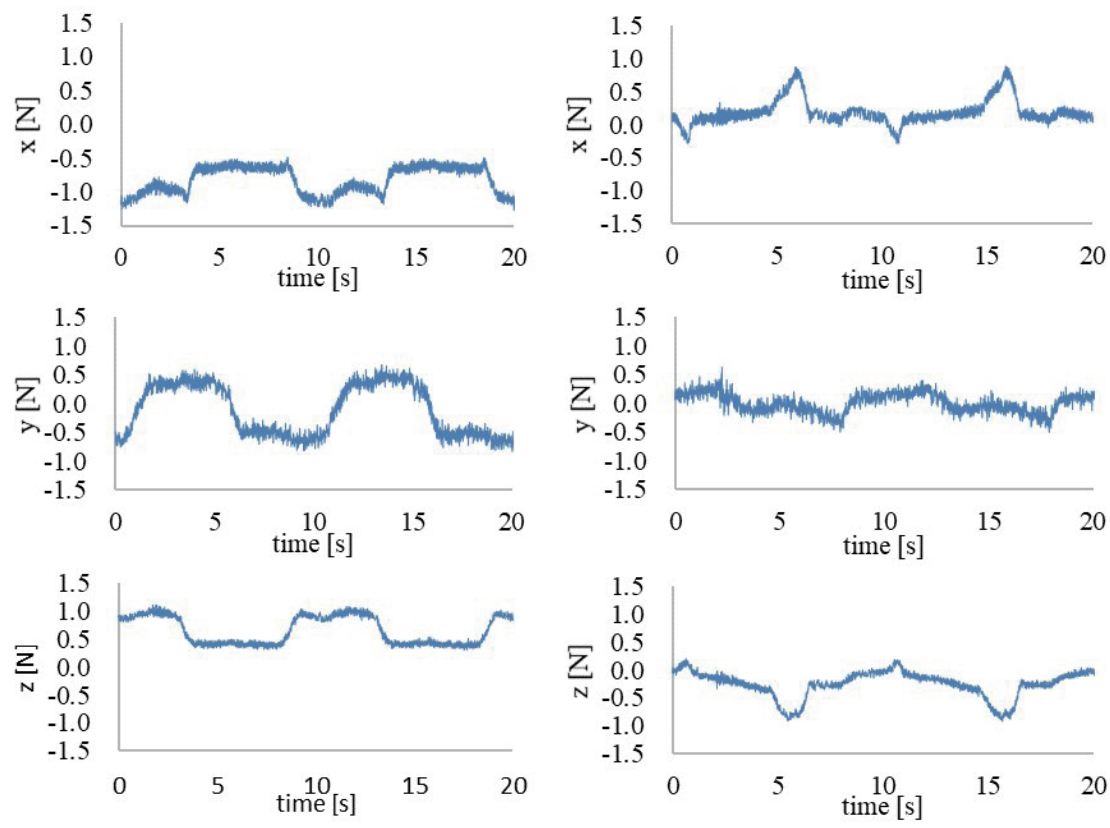

(a)

(b)

Fig. 12. (Color online) Forces exerted on the insertion port. (a) Passive joint and (b) Semi-active joint.

Table 2

Average magnitude of forces exerted on insertion port.

\begin{tabular}{lcc}
\hline & Passive joint $(\mathrm{N})$ & Semi-active joint $(\mathrm{N})$ \\
\hline$\left|F_{x}\right|$ & 0.82 & 0.21 \\
$\left|F_{y}\right|$ & 0.43 & 0.14 \\
$\left|F_{z}\right|$ & 0.65 & 0.27 \\
$|F|$ & 1.15 & 0.41 \\
\hline
\end{tabular}




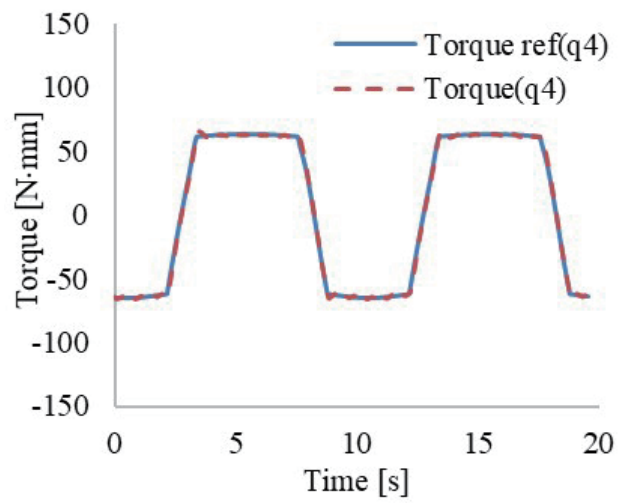

(a)

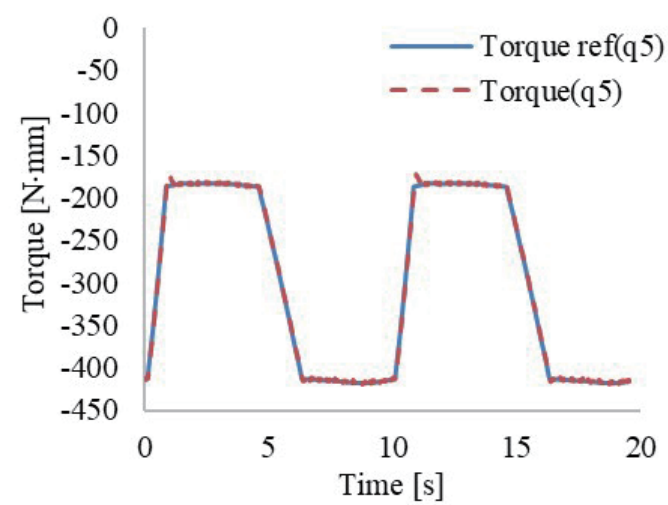

(b)

Fig. 13. (Color online) Output torques of semi-active joints. (a) $q_{4}$ and (b) $q_{5}$.

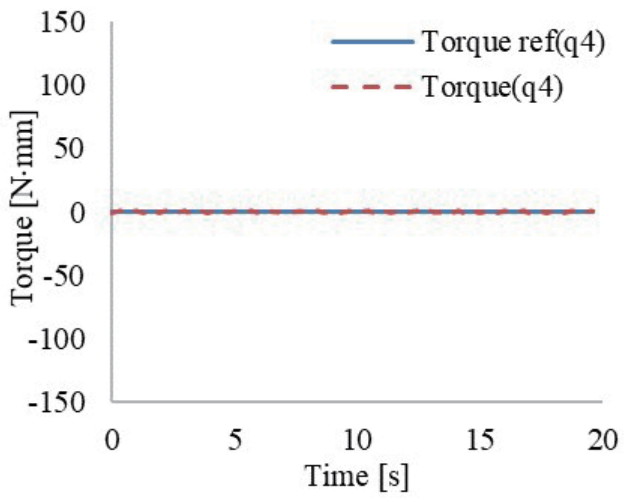

(a)

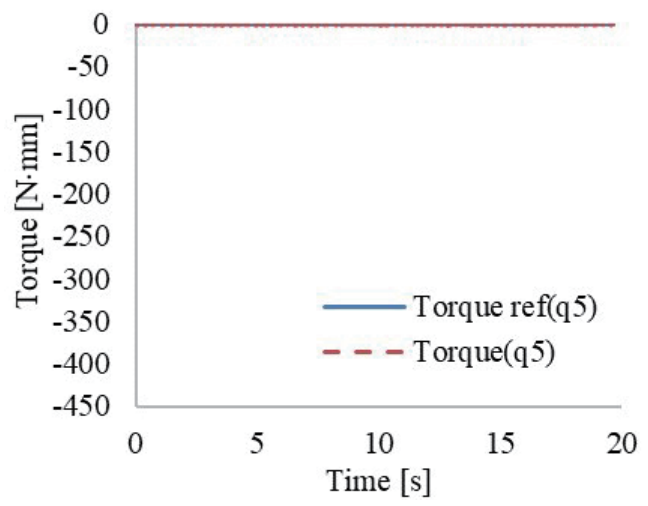

(b)

Fig. 14. (Color online) Output torques of passive joints. (a) $q_{4}$ and (b) $q_{5}$.

\section{Discussion}

\subsection{Reduction in forces exerted on insertion port with semi-active joints}

From the experimental results, it was confirmed that the proposed robotic arm can maintain its RCM passively and forces on the insertion port can be reduced by putting the semi-active joints on the robotic arm and driving suitable torque. Figure 12 shows how the forces of the forceps and mechanical impedance are compensated for using the semi-active joints. In Fig. 11, the reason why the followability to the target trajectory is better when controlled by semi-active joints is that the joints effectively compensate for the weight of the forceps. When the gimbal joints are passive and the weight of forceps is exerted on the insertion port, it is considered that the deviation from the target trajectory is caused by the backlash and deflection of the experimental device. The device has a hole as an insertion port, and the diameter of the hole is slightly larger than that of the forceps. Also, the gimbal of the port is cantilever-supported. The forceps can experience the backlash and deflection when strong forces are applied. Although 
we performed the experiment under ideal conditions, it can be said that the actual trocar and insertion ports are softer and more frictional. It is clear that it is better not to put a load on the insertion port.

In addition, as shown in Fig. 12, as compared with passive joints, semi-active joints could reduce the force on the insertion port from $1.15 \mathrm{~N}$ (passive) to $0.41 \mathrm{~N}$ (semi-active) on average. Then, Fig. 13(b) shows that joint $q_{5}$ outputs $300 \mathrm{~N} \cdot \mathrm{mm}$ torque on average. The distance from $q_{5}$ to its insertion port is about $300-400 \mathrm{~mm}$. Therefore, it is found that the output torque of the joints is valid. If the model of its weight is improved, the performance of forces exerted on the insertion port will be better.

\subsection{Impedance of insertion port}

In this study, the experimental device is an ideal insertion port that is fixed to the gimbal joint and does not move. However, a real insertion port on the abdomen may be soft and moved even by a slight force. An insertion port has impedance, which we did not consider. We have to conduct experiments on a soft insertion port.

\section{Conclusions}

To develop a surgical robotic arm that does not require precise alignment of its RCM and can compensate for external forces on the insertion port, we developed a robotic arm with semiactive joints. For these joints, we proposed a control method by which the robot maintains its RCM passively and compensates for forces on the insertion port. Then, we performed an experiment and evaluated the accuracy of the movement and forces on the insertion port. As a result, forces on the insertion port were reduced and the accuracy of the movement was improved by using semi-active joints.

In this study, however, the semi-active joints only compensate for the weight of the forceps and the dynamics of the arm and the forceps. A method to compensate for forces applied on the forceps is also required. Thus, we will develop a system that estimates external forces on the tip and compensates for them to reduce forces on the insertion port.

\section{References}

1 C. Freschi, V. Ferrari, F. Melfi, M. Ferrari, F. Mosca, and A. Cuschieri: Int. J. Med. Rob. Comput. Assisted Surg. 9 (2013) 396.

2 K. Tadano, K. Kawashima, K. Kojima, and N. Tanaka: J. Rob. Mechatron. 22 (2010) 179.

3 U. Hagn, M. Nickl, S. Jörg, G. Passig, T. Bahls, A. Nothhelfer, F. Hacker, L. Le-Tien, A. Albu-Schäffer, R. Konietschke, M. Grebenstein, R. Warpup, R. Haslinger, M. Frommberger, and G. Hirzinger: Ind. Robot 35 (2008) 324.

4 H. Sadeghian, F. Zokaei, and S.H. Jazi: J. Intell. Rob. Syst. (2018). https://doi.org/10.1007/s10846-018-0927-0

5 M. Ghodoussi, S. E. Butner, and Y. Wang: Proc. 2002 IEEE Int. Conf. Robotics and Automation (IEEE, 2002), 1882.

6 W. Wang, J. Li, S.Wang, H. Su, and X. Jiang: Int. J. Med. Rob. Comput. Assisted Surge 12 (2016) 73.

7 T. Ogawa and K. Tadano: Proc. Conf. Japan Fluid Power System Society (2016) 113 (in Japanese).

8 C. Gruijthuijsen, L. Dong, G. Morel, and E. V. Poorten: IEEE Rob. Autom. Lett. 3 (2018) 2071.

9 K. Kawashima, T. Sasaki, T. Miyata, N. Nakamura, M. Sekiguchi, and T. Kagawa: J. Rob. Mechatron. 16 (2004) 8. 


\section{About the Authors}

Koki Aizawa received his B.S. degree from Yokohama National University, Japan, in 2018. Since 2018, he has been a master's student at Tokyo Institute of Technology. His research interests are in robotics, mechanical engineering, and control engineering.

(aizawa.k.af@m.titech.ac.jp)

Masao Kanazawa received his B.S. and M.S. degrees from Tokyo Institute of Technology, Japan, in 2007 and 2009, respectively. From 2010 to 2018, he was engaged in developing humanoid robots at Honda R\&D Co., Ltd. Since 2018, he has been developing surgical robots at RIVERFIELD Inc., Japan. His research interests are in control engineering of surgical robots and humanoid robots. (m-kanazawa.rfc@riverfieldinc.com)

Daisuke Haraguchi received his B.S. degree from National Defence Academy, Japan, in 2003 and his M.S. and Ph.D. degrees from the Tokyo Institute of Technology, Japan, in 2010 and 2013, respectively. From 2014 to 2018, he was the CEO of RIVERFIELD Inc., Japan, developing and commercializing surgical robot products. Since 2019, he has been a research associate professor at Tokyo Institute of Technology. (haraguchi.d.aa@m.titech.ac.jp)

Kotaro Tadano received his B.S. degree in physics and M.S. and Dr. Eng. degrees in mechanical engineering from Tokyo Institute of Technology, Yokohama, Japan, in 2003, 2005, and 2007, respectively. He is currently an associate professor at Tokyo Institute of Technology, Yokohama, Japan. His research interests include robotics, teleoperation, and pneumatic systems. (tadano.k.aa@m.titech.ac.jp) 